\title{
The role of size constancy for the integration of local elements into a global shape
}

\author{
Johannes Rennig ${ }^{1}$, Hans-Otto Karnath ${ }^{1,2}$ and Elisabeth Huberle ${ }^{1,3 *}$ \\ ${ }^{1}$ Division of Neuropsychology, Center of Neurology, Hertie-Institute for Clinical Brain Research, University of Tübingen, Tübingen, Germany \\ 2 Department of Psychology, University of South Carolina, Columbia, SC, USA \\ ${ }^{3}$ Neurology and Neurorehabilitation Center, Luzerner Kantonsspital, Luzern, Switzerland
}

Edited by:

Magdalena Chechlacz, University of

Oxford, UK

Reviewed by:

Arnaud Saj, University Hospital of

Geneva, Switzerland

Celine R. Gillebert, University of

Oxford, UK

\section{*Correspondence:}

Elisabeth Huberle, Neurology and Neurorehabilitation Center, Luzerner Kantonsspital, Spitalstrasse,

CH-6000 Luzern 16, Switzerland e-mail: elisabeth.huberle@luks.ch

Visual perception depends on the visual context and is likely to be influenced by size constancy, which predicts a size and distance invariant perception of objects. However, size constancy can also result in optical illusions that allow the manipulation of the perceived size. We thus asked whether the integration of local elements into a global object can be influenced by manipulations of the visual context and size constancy? A set of stimuli was applied in healthy individuals that took advantage of the "Kanizsa" illusion, in which three circles with open wedges oriented toward a center point are placed to form an illusionary perception of a triangle. In addition, a 3D-perspective view was implemented in which the global target ("Kanizsa" triangle) was placed in combination with several distractor circles either in a close or a distant position. Subjects were engaged in a global recognition task on the location of the "Kanizsa" triangle. Global recognition of "Kanizsa" triangles improved with a decreasing length of the illusory contour. Interestingly, recognition of "Kanizsa" triangles decreased when they were perceived as if they were located further away. We conclude that the integration of local elements into a global object is dependent on the visual context and dominated by size constancy.

\section{Keywords: visual constancy, global perception, Gestalt, Kanizsa, visual context, perceptual grouping}

\section{INTRODUCTION}

Principles of Gestalt perception have strongly influenced our understanding of visual cognition. In the past century, Gestalt psychologists like Koffka (1935) and Wertheimer (1923) postulated that the human brain determines single elements with common features as a single entity rather than a sum of separate parts. Stimuli used to investigate the integration of multiple elements into a complex object can be found in Navon letters (Navon, 1977), i.e., hierarchically organized visual stimuli where a global letter is constructed from an array of local letters, or the "Kanizsa" illusion (Kanizsa, 1955). This illusion consists of an arrangement of circles with a wedge like gap (so called "pacman") that form an illusory angular figure. The "Kanizsa" illusion evokes neuronal responses similar to "real" geometrical figures in early and higher visual areas along the ventral stream (Hirsch et al., 1995; Ffytche and Zeki, 1996; Larsson et al., 1999; Halgren et al., 2003; Stanley and Rubin, 2003; Maertens and Pollmann, 2005, 2007; Maertens et al., 2008). Regarding the spatial characteristics of "Kanizsa" illusions it has been demonstrated that their global recognition performance decreases with an increasing length of the illusory contours (Kojo et al., 1993; Liinasuo et al., 1997). This observation is in line with a recent study showing that the spatial distance between local letters is crucial for recognition of Navon letters in neurological patients with simultanagnosia (Huberle and Karnath, 2006).

Besides physical properties of objects themselves, the visual environment plays an important role for efficient object recognition. In this context, the phenomenon of visual constancy is a crucial factor of human visual perception. Visual constancy is a key mechanism that allows the perception of familiar objects at a "standardized" shape, size, or color and is also critical for the invariant identification of objects regardless of changes in perspective, distance, lighting or the size of the retinal image (Emmert, 1881; Brunswik, 1934; Hebb, 1958; Fitzpatrick et al., 1982). Various perceptual illusions like the Ponzo or the MüllerLyer illusion (Müller-Lyer, 1889; Ponzo, 1911) are explained by size constancy-a crucial aspect of visual constancy enabling invariant size perception. Moore and Egeth (1997) revealed a preattentional influence for grouping mechanisms in the way that the length estimation of simple lines presented within a dot array was affected by the configuration of the dots in the background. When these dots formed a Ponzo or Müller-Lyer illusion, the length estimation changed depending on the arrangement of the surrounding dots. Importantly, the effect was present although the participants were unaware of the dot configurations in the background.

A previous study (Beck, 1975) demonstrated a significant role of perspective on global recognition performance. Global perception improved if the global target was perceived further away by tilting the stimulus; global recognition was supported by this perspective change which produced a closer retinal spacing between local elements. We therefore asked whether global recognition performance is dependent on the perceived distance between the individual elements of hierarchically organized stimuli and hypothesized that a perspective manipulation inducing larger distances between local elements by means of size constancy might 
result in decreased global recognition. In contrast to the work by Beck (1975), the present study aimed to achieve a distance manipulation by mechanisms of size constancy preserving a constant retinal image. In detail, we presented healthy observers with a $3 \mathrm{D}$ perspective view of an edged wall, in which an illusory "Kanizsa" triangle in an array of distractors was placed either at the close (Front condition) or the distant (Back condition) part of the wall. We assumed that the distant section of the wall would be perceived subjectively larger compared to the close section although the physical and retinal image remained unchanged. If size constancy influences Gestalt perception, the illusory contours of the "Kanizsa" triangles presented in the Back condition should be more difficult to perceive (cf. Beck, 1975), resulting in a decrease in recognition performance.

\section{METHODS \\ PARTICIPANTS}

Twenty healthy individuals ( 5 males, 15 females; average age 24.0 years, $S D=3.9)$ participated in Experiments 1 and 2; another 20 observers ( 6 males, 14 females; average age 25.5 years, $S D=4.4$ ) took part in Experiments 3 and 4; 22 subjects ( 2 males, 20 females; average age 23.4 years, $S D=4.1$ ) were tested in Experiment 5. All participants had normal or corrected-to-normal vision, no history of brain damage, and gave their informed consent before the participation in the study, which has been performed in accordance with the ethical standards laid down in the 1964 Declaration of Helsinki.

In Experiments 2 and 4, one participant had to be excluded for the final analysis due to low performance. Even in the easiest stimulus conditions where all other subjects showed an accuracy between $80-100 \%$, this subject's performance did not exceed the $50 \%$ chance rate. We attributed this behavior to a lack of motivation or comprehension. In Experiment 5, three participants had to be excluded due to the same reason.

\section{STIMULI}

\section{Experiment 1}

Stimuli displayed an edged stone wall with a perpendicular arrangement of the different parts of the wall (Figure 2A). The two main parts of the wall were oriented in parallel to the horizontal outline of the stimuli and appeared to be located close to the observer (Front) or further away (Back). In addition, the Front could be located to the left or the right from the center of the stimulus. All stimuli had a size of $19^{\circ} \times 19^{\circ}$ visual angle, in which the Front covered an area of $19.0^{\circ} \times 9.5^{\circ}$ with a size of the individual stones of $3.5^{\circ} \times 1.0^{\circ}$. The size of the Back was $7.0^{\circ} \times 5.0^{\circ}$ with a size of individual stones of $1.4^{\circ} \times 0.4^{\circ}$. A fixation dot was placed at the center of the stimulus.

Finally, a 3-2-3 array of white circles composed of wedge-like gaps (also known as "pacman") was superimposed on the wall. The size of the array was $4.0^{\circ} \times 4.0^{\circ}$. The center of this array was placed in the center of the Front or the Back and thus located $6.5^{\circ}$ to the right or the left from the central fixation dot. Three of the eight "pacmen" were oriented to enable the perception of an illusory triangle, while the remaining "pacmen" had a random orientation. The triangle was located at one of following positions within the array: (1) top/left, (2) top/right, (3) bottom/left, (4) bottom/right. The four possible positions were balanced for both presentation conditions (Front, Back) and sides (left, right) in all experiments. In order to facilitate the integration of the local "pacman" into a global triangle, we manipulated the size of the "pacmen." In detail, the following sizes of the "pacmen" were used: $0.4^{\circ}$ (Size 1), $0.5^{\circ}$ (Size 2), $0.6^{\circ}$ (Size 3), $0.7^{\circ}$ (Size 4), and $0.8^{\circ}$ (Size 5). The distance of $1.8^{\circ}$ (calculated from the center of the "pacmen") between the individual elements remained unchanged across conditions. Examples of the five sizes are shown in Figure 1.

\section{Experiment 2}

Experiment 2 aimed to test for differences in the integration of local elements into a global shape irrespective to the global perspective, but with respect to the local surround. The stimuli displayed a straight wall without the presence of edges (Figure 2B). The vertical extension of this wall was $11.0^{\circ}$, which was the average of the Front and Back in Experiment 1. In parallel to Experiment 1, the individual stones covered a size of $1.4^{\circ} \times 0.4^{\circ}$ (Back) or $3.5^{\circ} \times 1.0^{\circ}$ (Front). The stimulus parameters of the $3-2-3$ array were identical to Experiment 1.

\section{Experiment 3}

Experiment 3 aimed to test for differences in the integration of local elements into a global shape irrespective of the local surround, but with respect to the global perspective. The stimuli for Experiment 3 were comparable to the stimuli used for Experiment 1 with the exception that individual stones were replaced by a uniform gray surface (average of all pixels belonging to the stone wall in Experiment 1; Figure 3A). The 3D-perspective was generated by a squared pattern on the bottom in front of the wall.

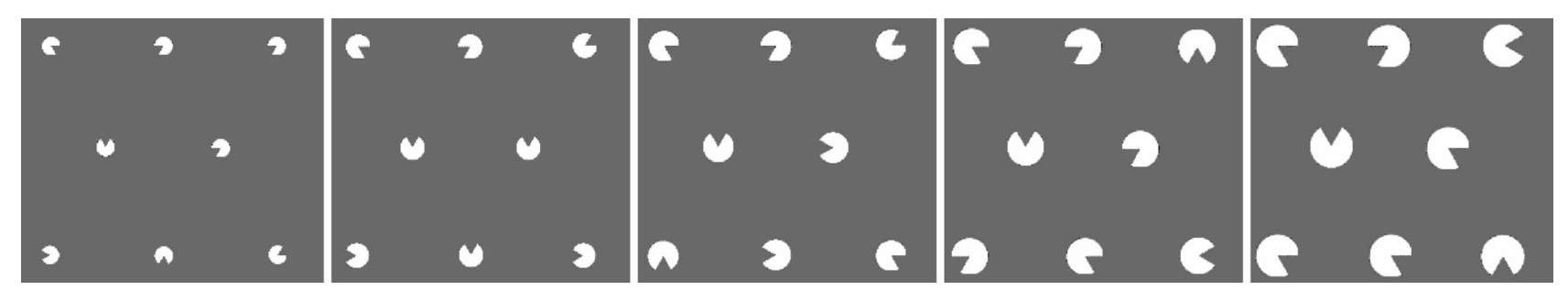

FIGURE 1 | Five 3-2-3 arrays with the five sizes of pacmen, with a Kanizsa triangle in the left upper corner; from left to right: Size 1-5. 


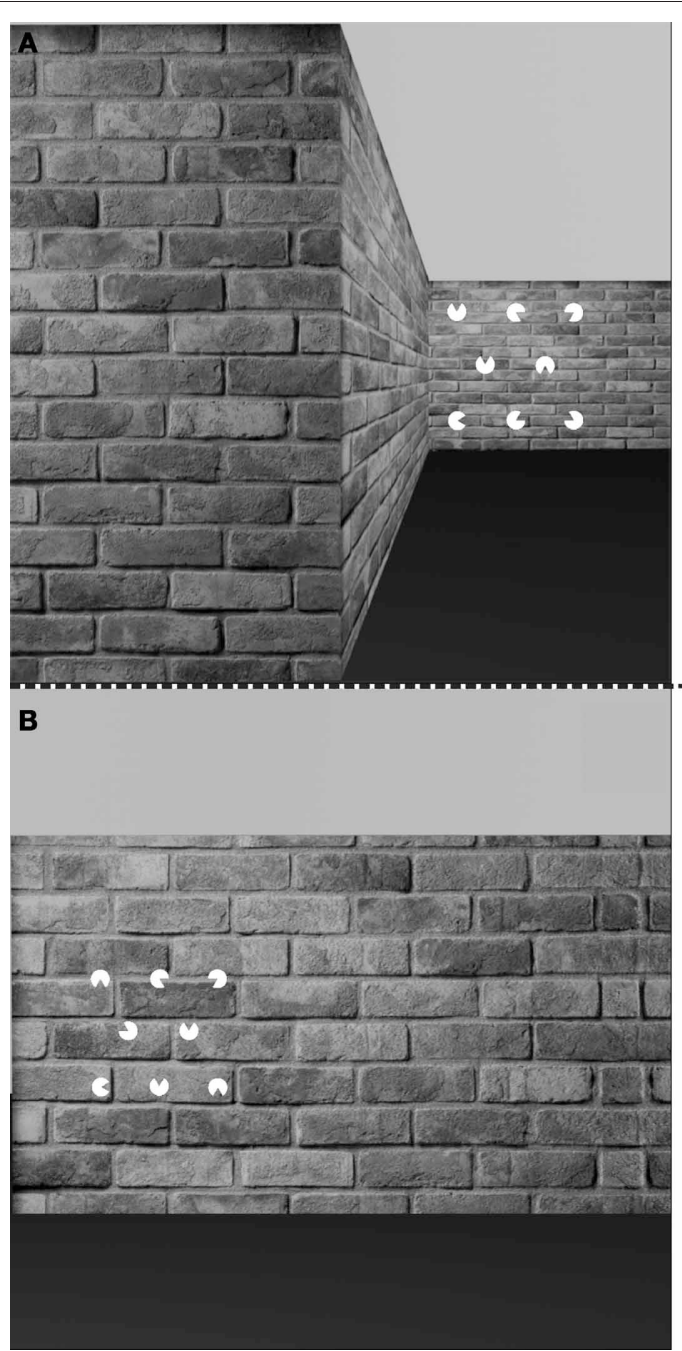

FIGURE 2 | Left: 3-2-3 array of white "pacmen" forming a "Kanizsa" triangle in one of four possible positions presented on one side of a shifted wall. (A) Stimulus for Experiment 1: 3-2-3 array presented on a naturalistic wall; (B) Stimulus for Experiment 2: 3-2-3 array presented on a wall appearing close (Front condition). The distant condition (Back) is not
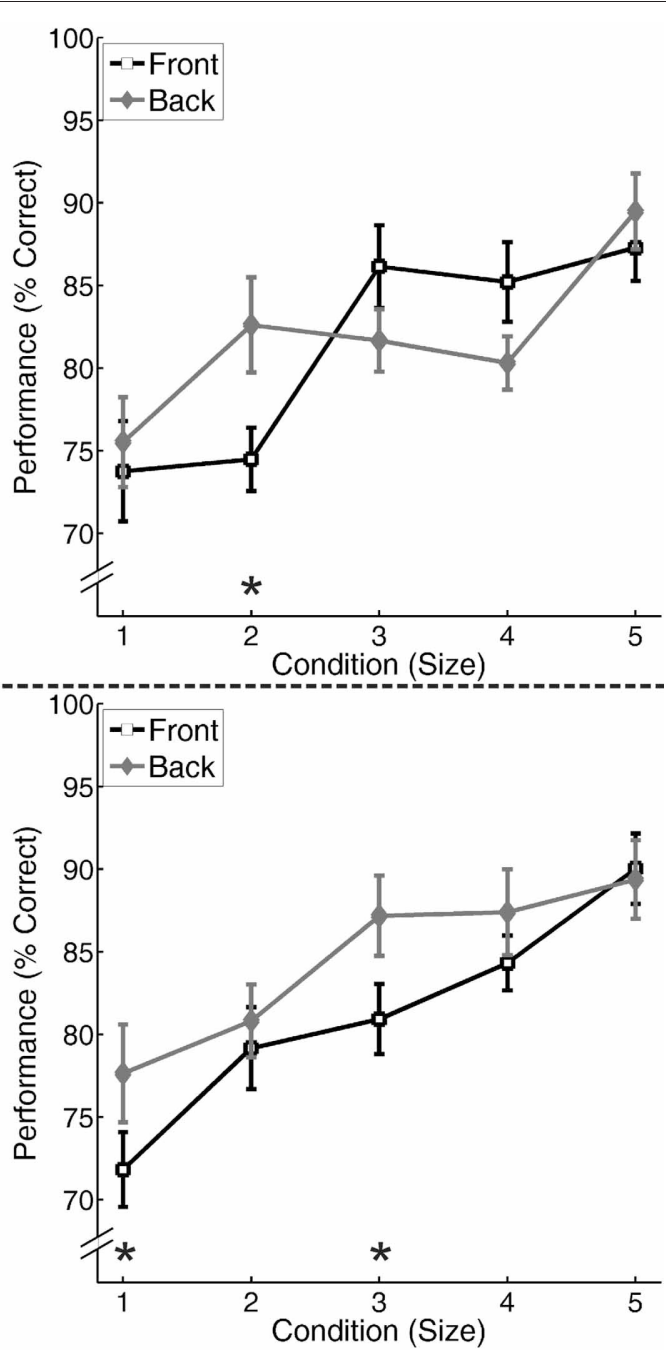

shown explicitly. Right: Results of Experiments 1 and 2 (A,B): displayed is the average percentage and standard errors of correct identification of "Kanizsa" triangles for the five different conditions of the "pacman" (Size $1-5)$ and two locations (Front, Back). ${ }^{*} p<0.01$, Bonferroni corrected for five comparisons.

\section{Experiment 4}

In parallel to Experiment 3, Experiment 4 aimed to test for differences in the integration of local elements into a global shape irrespective of the local surround, but with respect to the global perspective. The 3-2-3 array was embedded in a gray square (average of all pixels belonging to the stone wall in Experiment 1; Figure 3B) with a size of $4.8^{\circ} \times 5.4^{\circ}$, while the remaining stimulus parameters were identical to Experiment 1.

\section{Experiment 5}

Experiment 5 should control the possibility of an influence of local perspective effects by the size of the gray square used in Experiment 4. It consisted of two different parts: a preliminary test and the main experiment. In the preliminary test, the stimulus set of Experiment 4 was used but the 3-2-3 array was removed and a second gray square was added. In addition, the size of the gray square in the Back remained constant $\left(4.8^{\circ} \times 5.4^{\circ}\right)$ while the size of the square in the Front was presented in ten different sizes $\left(4.8^{\circ} \times 5.4^{\circ}, 5.0^{\circ} \times 5.6^{\circ}, 5.2^{\circ} \times 5.8^{\circ}, 5.4^{\circ} \times 6.0^{\circ}\right.$, $5.6^{\circ} \times 6.2^{\circ}, 5.8^{\circ} \times 6.4^{\circ}, 6.0^{\circ} \times 6.6^{\circ}, 6.2^{\circ} \times 6.8^{\circ}, 6.4^{\circ} \times 7.0^{\circ}$, $\left.6.6^{\circ} \times 7.2^{\circ}\right)$. Figure $3 \mathrm{C}$ shows a square of $6.4^{\circ} \times 7.0^{\circ}$, which was the size closest to the mean perceived size (see Results). We then determined the condition, in which the participant's perceived an equal size of the two gray squares. This condition was used for the main experiment, which was identical to Experiment 4.

\section{PROCEDURE}

All experiments were conducted in a room with dimmed light; stimuli were presented on a 19 inches CRT monitor with subjects located $57 \mathrm{~cm}$ in front of it. Stimulus presentation and data collection were controlled by a custom-made program using MatLab 


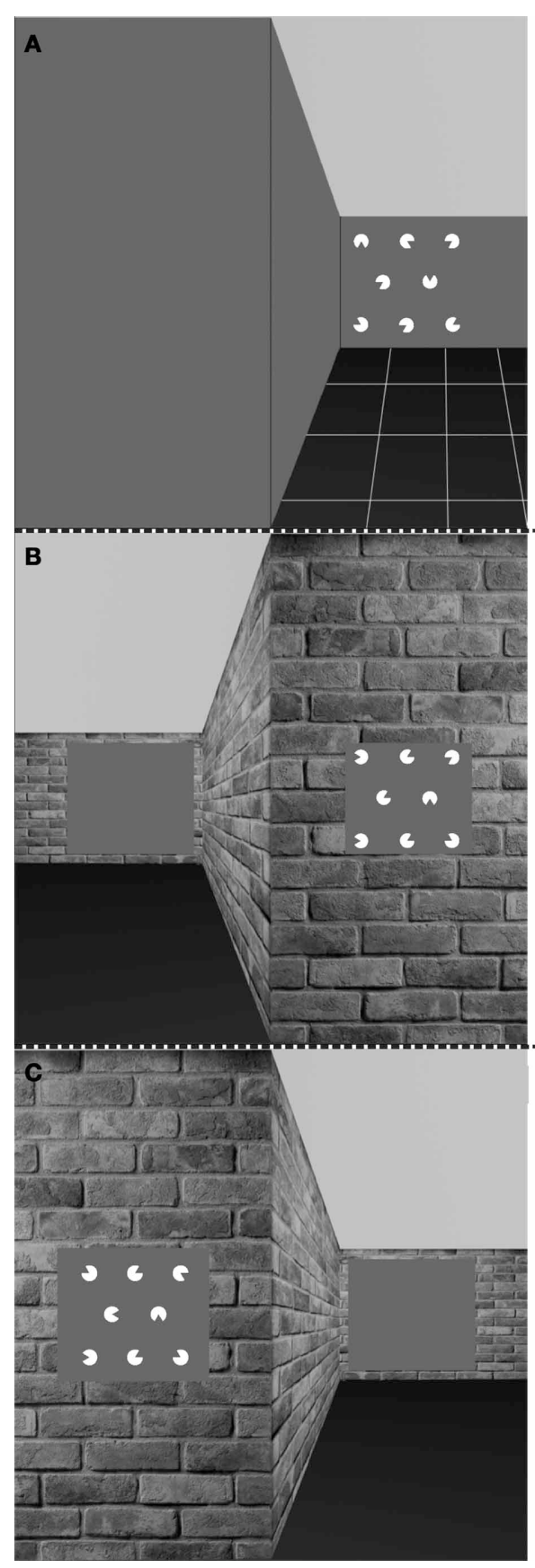

FIGURE 3 | Left: 3-2-3 array of white "pacmen" forming a "Kanizsa" triangle in one of four possible positions presented on one side of a shifted wall. (A) Stimulus for Experiment 3: 3-2-3 array presented on a uniform gray wall; (B) Stimulus for Experiment 4: 3-2-3 array presented on a gray box within a naturalistic wall. (C) Sample Stimulus for

Experiment 5: 3-2-3 array presented on a size adjusted gray box within a

2003b (MathWorks) and the Psychophysics Toolbox (Version 2.54; Brainard, 1997).

\section{Experiments 1-4}

The same design and procedure were used for Experiments 1-4. Each experiment consisted of ten conditions (Front: Size
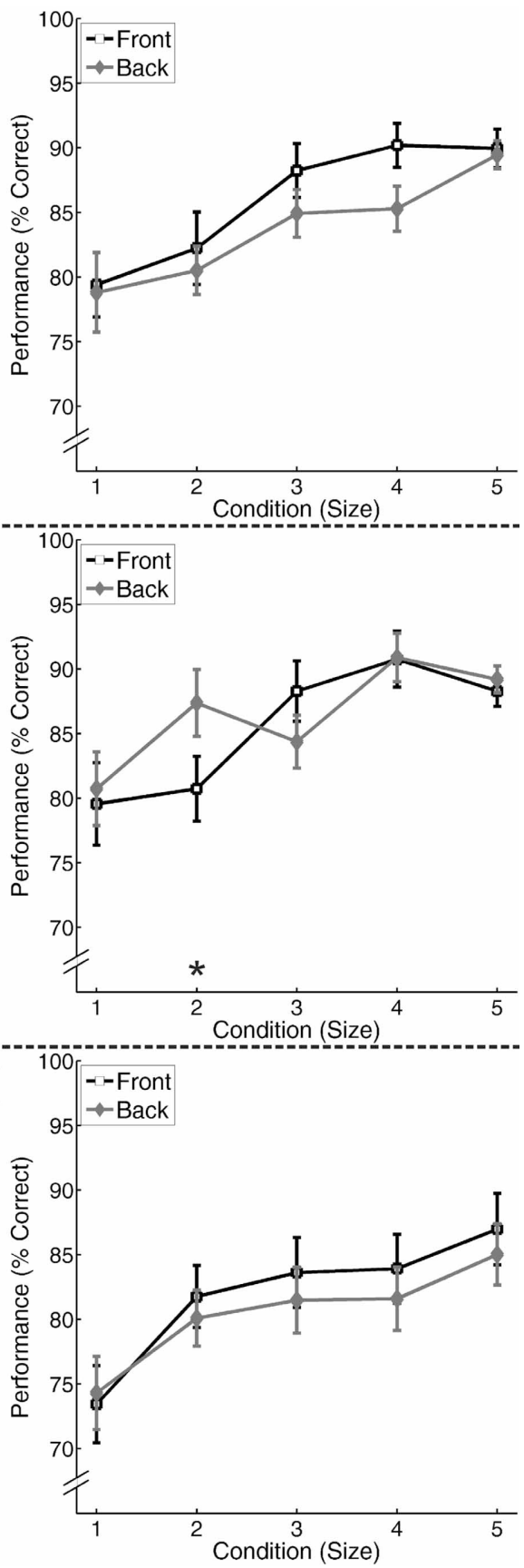

naturalistic wall $\left(6.4^{\circ} \times 7.0^{\circ}\right.$, which was the size closest to the mean perceived size). Right: Results of Experiments 3-5 (A-C): displayed is the average percentage and standard errors of correct identification of

"Kanizsa" triangles for the five different conditions of the "pacman" (Size 1-5) and two locations (Front, Back). ${ }^{*} p<0.01$, Bonferroni corrected for five comparisons.

1-5, Back: Size 1-5) that were repeated 48 times, resulting in a total number of 480 trials. All trials were presented in a random order in three blocks of 160 trials each. Before the onset of the first trial, all observers were familiarized with the type of stimuli and task in a short practice session. 
Each trial started with a stimulus presentation of $250 \mathrm{~ms}$ followed by a blank interval between 2250 and $3250 \mathrm{~ms}$, in which the central fixation dot was presented in a gray background. This procedure resulted in a trial duration between 2500 and $3500 \mathrm{~ms}$. During the blank interval, participants were engaged in a two alternative forced choice task on the position of the triangle. That is, they were instructed to indicate, if the location of the perceived triangle was at the top or the bottom of the 3-2-3 array by pressing a button in their right or left hand. The design was balanced for the position of the target triangle as well as the location of the array (left or right) with respect to its position (Front or Back). Further, the keymapping was counterbalanced across participants. Participants were instructed to maintain fixation throughout the study.

\section{Experiment 5}

The preliminary test consisted of ten conditions (Size 1-10) that were repeated 16 times each resulting in a total number of 160 trials. All trails were presented within one block. The participants were instructed to indicate which of the two squares was perceived larger. The condition of subjective equality was used for the main part of Experiment 5, which was identical to Experiments 1-4.

\section{RESULTS}

\section{Experiment 1}

Figure 2A shows that the percentage of correct responses tended to increase with an increasing size of the "pacman" for both Front and Back presentation conditions. We performed a repeatedmeasures ANOVA on the percentage of correct responses with Size (Size 1-5) and Position (Front, Back) as independent factors. The analysis revealed a significant interaction between the two factors $\left[F_{(4,16)}=4.50, p=0.01\right]$. Simple main effects were investigated by comparing Front and Back positions for every size of the "pacman" by paired T-tests. Using a Bonferroni-correction to correct for multiple comparisons (resulting in an alpha level of.01) we observed significant differences for Size 2 [2: $T_{(19)}=$ 3.63, $p=0.002]$, but not for Sizes 1 and 3-5 [1: $T_{(19)}<1, p=$ $0.41 ; 3: T_{(19)}=-2.20, p=0.041 ; 4: T_{(19)}=-2.48, p=0.023$; 5: $\left.T_{(19)}=1.41, p=0.18\right]$. Bonferroni-corrections regarding the post-hoc tests were applied for all conducted experiments.

\section{Experiment 2}

In parallel to Experiment 1, the percentage of correct responses showed a clear tendency to increase with an increasing size of the "pacman," again for both presentation conditions (see Figure 2B). In parallel to Experiment 1, we conducted a repeated-measures ANOVA with the same factors and observed a significant interaction between these factors $\left[F_{(4,15)}=27.85\right.$, $p<0.001]$ as well as a significant difference between the two positions for Sizes 1 and 3 [1: $T_{(18)}=5.59, p<0.001 ; 3: T_{(18)}=3.67$, $p=0.002]$, but not for 2 , 4 , and $5\left[2: T_{(18)}=1.32, p=0.21 ; 4\right.$ : $\left.T_{(18)}=1.76, p=0.10 ; 5: T_{(18)}<1, p=0.61\right]$.

\section{Experiment 3}

Performance was higher, if the 3-2-3 array was presented in the Front and tended to increase with an increasing size of the "pacman" for both presentation conditions (see Figure 3A). The repeated measures ANOVA with Size and Position as independent factors revealed a significant main effect for Size $\left[F_{(4,13)}=\right.$ 10.81, $p<0.001]$ and Position $\left[F_{(1,16)}=9.81, p=0.006\right]$ but no significant interaction $\left[F_{(4,13)}=1.38, p=0.30\right]$.

\section{Experiment 4}

Performance tended to increase with an increasing size of the "pacman" (Figure 3B). The repeated-measures ANOVA revealed a significant interaction between the two factors $\left[F_{(4,13)}=4.58\right.$, $p=0.02]$. By comparing Front and Back positions with paired $T$-tests for each size, we revealed significant differences for Size $2\left[T_{(15)}=3.34, p=0.004\right]$, but not for Sizes 1, 3-5 [1: $T_{(15)}<$ $1, p=0.45 ; 3: T_{(15)}=-2.05, p=0.60 ; 4: T_{(15)}<1, p=0.91$; 5: $\left.T_{(15)}<1, p=0.48\right]$.

\section{Experiment 5}

Also in this experiment, performance showed a clear tendency to increase with an increasing size of the "pacman" (see Figure 3C). Two squares were perceived equally large if the square in the Front condition was $41 \%$ larger than in the Back. With the adjusted square, performance again increased with an increasing size of the "pacman" (see Figure 3C). For the main experiment, the repeated measures ANOVA revealed a significant main effect for Size $\left[F_{(4,14)}=9.61, p=0.001\right]$, but not for Position $\left[F_{(1,17)}=\right.$ 2.97, $p=0.13]$. The interaction effect was also not significant $\left[F_{(4,14)}<1, p=0.70\right]$.

\section{Comparison experiment 1 vs. 3}

To directly test for effects of local details, we performed a three-way ANOVA with Size (1-5), Position (Front, Back) and Experiment (1 and 3 ) as independent factors. This ANOVA revealed a significant three-way interaction of Size, Position and Experiment: $\left[F_{(4,34)}=8.05, p<0.001\right]$ that strengthens the assumption for a crucial role of the visual context regarding effects of visual constancy in visual integration.

\section{DISCUSSION}

The present study aimed to investigate the role of size constancy for global recognition in a task requiring the integration of local elements into a global object. We took advantage of the "Kanizsa" illusion while size constancy was achieved by a 3D-perspective in which the "Kanizsa" illusion was placed. According to previous observations emphasizing the length of the illusory contours as a crucial factor for the perception of "Kanizsa" triangles (Kojo et al., 1993; Liinasuo et al., 1997), we assumed that global recognition performance was lower if the local elements were perceived to be further away from each other (Beck, 1975). A general decrease in recognition performance was thus assumed for the Back compared to the Front. In line with these predictions is the data of Experiment 3, demonstrating lower global recognition performance of the target object in the Back than in the Front condition. Noteworthy, in this experiment only global information of the visual background was available, while local information (individual stones, texture) was removed. A similar but not significant pattern was observed in Experiment 5 after the size of the gray square was adjusted and thus equally large perceived in the Front and Back. 
In this context, data from patients with simultanagnosia, a rare neurological disorder describing the inability to perceive a global Gestalt (Bálint, 1909), should be noted: global recognition performance in simultanagnosics can be modulated by the spatial distance between local elements and improves with smaller distances between elements (Huberle and Karnath, 2006). Further work indicated a key role of the visual angle together with the retinal image for global object recognition (Huberle et al., 2010). It could be demonstrated that rather the retinal image than the physical size of an object has a major impact on global perception. In addition, saliency has been linked to global recognition performance in simultanagnosics (Huberle and Karnath, 2010). However, the current results extent previous work and indicated that the perceived size might be more important for the recognition of a global Gestalt than its physical size. Further, the present findings cannot simply be attributed to retinal images and saliency, which were identical between the Front and Back condition. The explanation might rather be found in a systematic effect of size constancy on global perception that is attributed to the limited amount of local context information immediately available to the observer. In case of the absence of local context information a pronounced effect of size constancy on global perception is evident. Size constancy underlies the observation that separate objects presented in an enriched visual context are rather perceived at a subjective size. A recent study investigating size constancy in a virtual reality environment demonstrated that despite of an identical physical and retinal image perceived object size was mainly determined by perspective manipulations (Kenyon et al., 2008).

The results of the remaining experiments indicated a more complex interaction between size constancy and the integration of local elements into a global object. First, the data of Experiment 1 suggested an influence of local context information, namely the stone-like surface used for the wall. If the "pacmen" were large, the results were similar to Experiments 3 and 5. However, for small sizes of the "pacmen" the reversed pattern-higher global recognition performance in the Back than in the Front-was observed. Similar results became evident for Experiment 4. The differences in the global recognition performance between Experiments 4 and 5 might also be explained by local context information. In Experiment 5, the gray square in the Front condition was more than 40\% larger than in Experiment 4 and therefore covered more of the local surround. Moreover, the stimulus presentation of Experiment 3 is comparable to earlier work by Beck (1975), where global stimuli were also presented on a plane background. Further, local context information should also be regarded together with visual crowding, the interaction between nearby contours or "visual clutter" on visual discrimination and object recognition (Levi, 2008; Pelli and Tillman, 2008). "Visual clutter" surround global targets might disturb global recognition performance (Dakin and Baruch, 2009; Kingdom and Prins, 2009; Lau and Cheung, 2012; Robol et al., 2012). The idea of an interaction with local context information was further strengthened by the results of Experiment 2. Local context information with a minimized global surround (flat wall with small and large stones) showed a lower global recognition performance if large stones (equal to the Front) were used instead of smaller ones (similar to the Back). Moreover, the results of this experiment suggest that global recognition performance in the remaining experiments was mainly influenced by perspective manipulations and was independent from local context information. However, the present data cannot answer the question if local details changed the perceived size between local elements or influenced visual integration in general. Evidence about visual crowding and object perception (Levi, 2008; Pelli and Tillman, 2008), nevertheless, suggests a general influence of local details on integration processes. These observations reported here were mainly restricted to Sizes 2-4 while differences between Back and Front presentation for Sizes 1 and 5 were less pronounced over all experiments. We address this effect to a higher experimental sensitivity for the middle sizes of the stimulus spectrum. Moreover, missing variability for Size 5 can be attributed to a general ceiling effect for the 'easiest' condition, while Sizes 2-4 appeared to be more sensitive to reveal perspective differences between the Back and Front presentation.

Recent work observed a faster recognition of meaningful arrays creating illusory contours compared to random configurations of local elements in a noisy visual background indicating also an involvement of early visual areas in integration mechanisms (Wang et al., 2012). Further, neuroimaging studies have identified distinct neuronal networks of illusory contour processing (Hirsch et al., 1995; Ffytche and Zeki, 1996; Halgren et al., 2003; Maertens and Pollmann, 2005, 2007). However, the evidence of influences of size constancy on Gestalt perception supports the view of contributions of later visual areas to processes of visual integration. Various studies showed that functions of object perception get affected by size constancy (Emmert, 1881; Fitzpatrick et al., 1982; Kenyon et al., 2008) and localized object processing beyond early visual areas along the ventral visual pathway (Ungerleider and Mishkin, 1982; Goodale and Milner, 1992; Grill-Spector, 2003). Therefore, the presented results demonstrating influences of size constancy on Gestalt perception are in line with various findings from patients with simultanagnosia and healthy subjects attributing global perception to posterior parietal brain areas (Rizzo and Hurtig, 1987; Friedman-Hill et al., 1995; Rafal, 1997; Karnath et al., 2000; Tang-Wai et al., 2004; Valenza et al., 2004; Himmelbach et al., 2009; Huberle and Karnath, 2012; Thomas et al., 2012).

The current results suggest an important role of size constancy on global perception of hierarchically organized visual stimuli. In accordance with previous findings, the distance between local elements in illusory global objects composed of local elements appears to be a crucial factor for global recognition. Emphasizing the importance of the perceived size of a global figure in visual integration, the data extend previous findings in patients with simultanagnosia that attributed global recognition performance to the retinal size rather than the physical. To our knowledge, the work shown here represents the first manipulation of global recognition with mechanisms of size constancy preserving a constant retinal image and, thus, highlights the role of the perceived 
size of a global object in the process of visual integration. Size constancy influences our perception and leads to an enlarged inter-element spacing in hierarchically organized global figures. Finally, local context information shows an interaction with size constancy.

\section{REFERENCES}

Bálint, R. (1909). Seelenlähmung des "Schauens", optische Ataxie, räumliche Störung der Aufmerksamkeit. Mschr. Psychiat. Neurol. 25, 51-81.

Beck, J. (1975). The relation between similarity grouping and perceptual constancy. Am. J. Psychol. 88, 397-409. doi: 10.2307/ 1421770

Brainard, D. H. (1997). The psychophysics toolbox. Spat. Vis. 10, 433-436. doi: 10.1163/156856897 X00357

Brunswik, E. (1934). Wahrnehmung und Gegenstandswelt: Grundlegung einer Psychologie vom Gegenstand her. Leipzig: Deuticke.

Dakin, S. C., and Baruch, N. J. (2009). Context influences contour integration. J. Vis. 9, 13.1-13.13.

Emmert, E. (1881). Grössenverhältnisse der Nachbilder. Klin. Monatsbl. Augenheilkd. 443-450.

Ffytche, D. H., and Zeki, S. (1996). Brain activity related to the perception of illusory contours. Neuroimage 3, 104-108. doi: 10.1006/nimg.1996.0012

Fitzpatrick, V., Pasnak, R., and Tyer, Z. E. (1982). The effect of familiar size at familiar distances. Perception 11, 85-91. doi: 10.1068/ p110085

Friedman-Hill, S. R., Robertson, L. C., and Treisman, A. (1995). Parietal contributions to visual feature binding: evidence from a patient with bilateral lesions. Science 269, 853-855. doi: $10.1126 /$ science. 7638604

Goodale, M. A., and Milner, A. D. (1992). Separate visual pathways for perception and action. Trends Neurosci. 15, 20-25. doi: 10.1016/0166-2236 (92)90344-8

Grill-Spector, K. (2003). The neural basis of object perception. Curr. Opin. Neurobiol. 13, 159-166. doi: 10.1016/S0959-4388 (03)00040-0

Halgren, E., Mendola, J., Chong, C. D. R., and Dale, A. M. (2003). Cortical activation to illusory shapes as measured with magnetoencephalography.
Neuroimage 18, 1001-1009. doi: 10.1016/S1053-8119(03)00045-4

Hebb, D. O. (1958). Textbook of Psychology. Philadelphia: W.B. Saundery Company. doi: 10.1037/14200-000

Himmelbach, M., Erb, M., Klockgether, T., Moskau, S., and Karnath, H. O. (2009). fMRI of global visual perception in simultanagnosia. Neuropsychologia 47, 1173-1177. doi: 10.1016/ j.neuropsychologia.2008.10.025

Hirsch, J., DeLaPaz, R. L., Relkin, N. R., Victor, J., Kim, K., Li, T., et al. (1995). Illusory contours activate specific regions in human visual cortex: evidence from functional magnetic resonance imaging. Proc. Natl. Acad. Sci. U.S.A. 92, 6469-6473. doi: 10.1073/pnas.92.14.6469

Huberle, E., Driver, J., and Karnath, H. O. (2010). Retinal versus physical stimulus size as determinants of visual perception in simultanagnosia. Neuropsychologia 48, 1677-1682. doi: 10.1016/ j.neuropsychologia.2010.02.013

Huberle, E., and Karnath, H. O. (2006). Global shape recognition is modulated by the spatial distance of local elements-evidence from simultanagnosia. Neuropsychologia 44, 905-911. doi: 10.1016/ j.neuropsychologia.2005.08.013

Huberle, E., and Karnath, H. O. (2010). Saliency modulates global perception in simultanagnosia. Exp. Brain Res. 204, 595-603. doi: 10.1007/s00221-0102328-x

Huberle, E., and Karnath, H. O. (2012). The role of temporo-parietal junction (TPJ) in global Gestalt perception. Brain Struct. Funct. 217, 735-746. doi: 10.1007/s00429-0110369-y

Kanizsa, G. (1955). Quasi-perceptional margins in homoge- nously stimulated fields. Psicologia 49, 7-30.

Karnath, H., Ferber, S., Rorden, C., and Driver, J. (2000). The fate of global information in dorsal simultanagnosia. Neurocase 6, 295-306. doi: 10.1080/13554790008402778

Kenyon, R. V., Phenany, M., Sandin, D., and Defanti, T. (2008)

\section{ACKNOWLEDGMENTS}

This work was supported by a grant of the Bundesministerium für Bildung und Forschung (BMBF-Verbund 01GW0654 "Visuospatial cognition") the Deutsche Forschungsgemeinschaft (DFG) and the Open Access Publishing Fund of the University of Tübingen. We thank Maren Prass for her helpful discussion.

Accommodation and sizeconstancy of virtual objects. Ann. Biomed. Eng. 36, 342-348. doi: 10.1007/s10439-007-9414-7

Kingdom, F. A. A., and Prins, N. (2009). Texture-surround suppression of contour-shape coding in human vision. Neuroreport 20, 5-8. doi: 10.1097/WNR.0b013e32831578ca

Koffka, K. (1935). Principles of Gestalt Psychology. 1st Edn. New York, NY: Harcourt.

Kojo, I., Liinasuo, M., and Rovamo, J. (1993). Spatial and temporal properties of illusory figures. Vision Res. 33, 897-901. doi: 10.1016/0042-6989 (93)90072-5

Larsson, J., Amunts, K., Gulyás, B., Malikovic, A., Zilles, K., and Roland, P. E. (1999). Neuronal correlates of real and illusory contour perception: functional anatomy with PET. Eur. J. Neurosci. 11, 4024-4036. doi: 10.1046/j.14609568.1999.00805.x

Lau, J. S. F., and Cheung, S. (2012) Illusory contour formation survives crowding. J. Vis. 12, 15. doi $10.1167 / 12.6 .15$

Levi, D. M. (2008). Crowding-an essential bottleneck for object recognition: a mini-review. Vision Res. 48, 635-654. doi: 10.1016/ j.visres.2007.12.009

Liinasuo, M., Rovamo, J., and Kojo, I. (1997). Effects of spatial configuration and number of fixations on Kanizsa triangle detection. Invest. Ophthalmol. Vis. Sci. 38, 2554-2565.

Maertens, M., and Pollmann, S. (2005). fMRI reveals a common neural substrate of illusory and real contours in V1 after perceptual learning. J. Cogn. Neurosci. 17, 1553-1564. doi: 10.1162/0898 92905774597209

Maertens, M., and Pollmann, S. (2007). Illusory contours do not pass through the "blind spot". J. Cogn. Neurosci. 19, 91-101. doi: 10.1162/jocn.2007.19.1.91

Maertens, M., Pollmann, S., Hanke, M., Mildner, T., and Möller, $\mathrm{H}$. (2008). Retinotopic activation in response to subjective contours in primary visual cortex. Front. Hum.
Neurosci. 2:2. doi: 10.3389/neuro.09. 002.2008

Moore, C. M., and Egeth, H. (1997) Perception without attention: evidence of grouping under conditions of inattention. J. Exp. Psychol. Hum. Percept. Perform. 23, 339-352. doi: 10.1037/00961523.23.2.339

Müller-Lyer, F. C. (1889). Optische Urteilstäuschungen. Arch. Physiol. 263-270.

Navon, D. (1977). Forest before trees: the precedence of global features in visual perception. Cogn. Psychol. 383, 353-383. doi: 10.1016/00100285(77)90012-3

Pelli, D. G., and Tillman, K. A. (2008). The uncrowded window of object recognition. Nat. Neurosci. 11, 1129-1135. doi: 10.1038/nn.2187

Ponzo, M. (1911). Intorno ad alcune illusioni nel campo delle sensazioni tattili sull'illusione di Aristotele e fenomeni analoghi. Arch. Gesamte Psychol. 16, 307-345.

Rafal, R. (1997). "Balint syndrome," in Behavioral Neurology and Neuropsychology, eds T. Feinberg and M. Farah (Boston, MA McGraw-Hill), 337-356.

Rizzo, M., and Hurtig, R. (1987). Looking but not seeing: attention, perception, and eye movements in simultanagnosia. Neurology 37, 1642-1648. doi: 10.1212/WNL.37.10.1642

Robol, V., Casco, C., and Dakin, S. C. (2012). The role of crowding in contextual influences on contour integration. J. Vis. 12, 3. doi: 10.1167/12.7.3

Stanley, D. A., and Rubin, N. (2003). fMRI activation in response to illusory contours and salient regions in the human lateral occipital complex. Neuron 37, 323-331. doi: 10.1016/S0896-6273 (02)01148-0

Tang-Wai, D. F., Graff-Radford, N. R., Boeve, B. F., Dickson, D. W. Parisi, J. E., Crook, R., et al. (2004). Clinical, genetic, and neuropathologic characteristics of posterior cortical atrophy. Neurology 63, 1168-1174. doi: 10.1212/ 01.WNL.0000140289.18472.15 
Thomas, C., Kveraga, K., Huberle, E., Karnath, H. O., and Bar, M. (2012). Enabling global processing in simultanagnosia by psychophysical biasing of visual pathways. Brain 135, 1578-1585. doi: 10.1093/brain/ aws066

Ungerleider, L., and Mishkin, M. (1982). "Two cortical visual systems," in Analysis of Visual Behavior, eds D. J. Ingle, M. A. Goodale, and R. J. E. Mansfield (Cambridge, MA: MIT Press), 549-586.
Valenza, N., Murray, M. M., Ptak, R., and Vuilleumier, P. (2004). The space of senses: impaired crossmodal interactions in a patient with Balint syndrome after bilateral parietal damage. Neuropsychologia 42, 1737-1748. doi: 10.1016/ j.neuropsychologia.2004.05.001

Wang, L., Weng, X., and $\mathrm{He}$, S. (2012). Perceptual grouping without awareness: superiority of Kanizsa triangle in breaking interocular suppression. PLoS ONE 7:e40106. doi: 10.1371/journal. pone.0040106
Wertheimer, M. (1923). Untersuchungen zur Lehre von der Gestalt. Psychol. Forsch. 301-350.

Conflict of Interest Statement: The authors declare that the research was conducted in the absence of any commercial or financial relationships that could be construed as a potential conflict of interest.

Received: 29 April 2013; accepted: 17 June 2013; published online: 03 July 2013.
Citation: Rennig J, Karnath $\mathrm{H}-\mathrm{O}$ and Huberle E (2013) The role of size constancy for the integration of local elements into a global shape. Front. Hum. Neurosci. 7:342. doi: 10.3389/fnhum. 2013.00342

Copyright (๑) 2013 Rennig, Karnath and Huberle. This is an open-access article distributed under the terms of the Creative Commons Attribution License, which permits use, distribution and reproduction in other forums, provided the original authors and source are credited and subject to any copyright notices concerning any third-party graphics etc. 